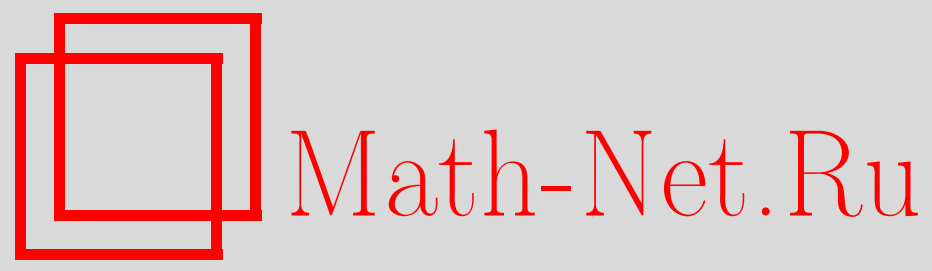

В. Г. Михайлов, Об асимптотических свойствах распределения числа пар $H$-связанных цепочек, Дискрет. матем., 2002, том 14, выпуск 3, 122-129

DOI: https://doi.org/10.4213/dm259

Использование Общероссийского математического портала Math-Net.Ru подразумевает, что вы прочитали и согласны с пользовательским соглашением http: //www . mathnet.ru/rus/agreement

Параметры загрузки:

IP : 54.224 .187 .69

26 апреля 2023 г., 16:50:24

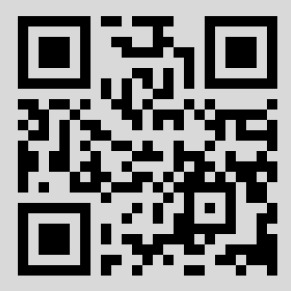




\title{
Об асимптотических свойствах распределения числа пар $H$-связанных цепочек
}

\author{
() 2002 г. В. Г. Михайлов
}

\begin{abstract}
Основной результат статьи - теорема о сходимости к пуассоновскому распределению распределения числа пар $H$-связанных $s$-цепочек в двух независимых последовательностях независимых одинаково распределенных величин. Понятие $H$-связанности обобщает использованное рядом авторов понятие $H$-эквивалентности цепочек. Указаны достаточные условия сходимости, получена явная оценка скорости сходимости. При доказательстве использован локальный вариант метода Чена-Стейна оценивания точности пуассоновской аппроксимации для распределения набора зависимых случайных индикаторов.

Работа выполнена при поддержке Российского фонда фундаментальных исследований, проект 02-01-00266, и Совета по грантам Президента РФ и государственной поддержке ведущих научных школ, проект 00-15-96136.
\end{abstract}

Пусть $X=\left(X_{1}, \ldots, X_{m}\right)$ и $Y=\left(Y_{1}, \ldots, Y_{n}\right)$ - две последовательности независимых одинаково (внутри каждой последовательности) распределенных случайных величин, принимающих значения в множестве $\{1,2, \ldots\}$,

$$
\begin{aligned}
& p_{k}=\mathbf{P}\left\{X_{1}=k\right\}, \quad q_{k}=\mathbf{P}\left\{Y_{1}=k\right\}, \quad k=1,2, \ldots, \\
& p=\max _{k} p_{k}, \quad q=\max _{k} q_{k}, \quad P=m p^{s}, \quad Q=n q^{s} .
\end{aligned}
$$

Пусть задан некоторый конечный набор $H$ взаимно однозначных отображений

$$
h:\{1,2, \ldots\} \rightarrow\{1,2, \ldots\} .
$$

Две цепочки $\left(x_{1}, \ldots, x_{s}\right)$ и $\left(y_{1}, \ldots, y_{s}\right)$ знаков алфавита $\{1,2, \ldots\}$ будем называть $H$-связанными, если найдется подстановка $h \in H$, для которой

$$
y_{i}=h\left(x_{i}\right), \quad i=1, \ldots, s .
$$

В этом определении было бы естественным считать, что $\{e\} \in H$ (e - тождественная подстановка) и $h^{-1} \in H$ для всех $h \in H$. Однако все утверждения и доказательства настоящей работы справедливы без этих предположений, поэтому мы их не используем.

Введем обозначения

$$
\begin{aligned}
E(i, j ; h) & =\left\{\left(h\left(X_{i}\right), \ldots, h\left(X_{i+s-1}\right)\right)=\left(Y_{j}, \ldots, Y_{j+s-1}\right)\right\}, \\
E(i, j) & =\bigcup_{h \in H} E(i, j ; h) .
\end{aligned}
$$


Число пар $H$-связанных цепочек из последовательностей $X$ и $Y$ выражается формулой

$$
N(s, m, n)=\sum_{i=1}^{m} \sum_{j=1}^{n} I(E(i, j))
$$

(здесь и далее выражение $I(\cdot)$ обозначает индикатор случайного события или неслучайного множества). В этом определении считается, что

$$
X_{m+i}=X_{i}, \quad Y_{n+i}=Y_{i}, \quad i=1,2, \ldots
$$

Это предположение позволяет отчасти упростить выкладки, но не влияет на вид предельного распределения.

Рассмотрим случай, когда с ростом $m, n$ параметры $p_{k}, q_{k}, s$ меняются так, что математическое ожидание случайной величины $N(s, m, n)$ сходится к некоторому конечному пределу.

Введем обозначение

$$
D_{k}=\mathbf{P}\{E(1,1) E(1+k, 1+k)\}, \quad k=0,1, \ldots, s-1 .
$$

В частности,

$$
D_{0}=\mathbf{P}\{E(1,1) E(1,1)\}=\mathbf{P}\{E(1,1)\}
$$

Пусть

$$
D=D_{1}+\ldots+D_{s-1}
$$

Распределение Пуассона с параметром $\lambda$ условимся обозначать Ро $(\lambda)$.

Теорема 1. Пусть параметры схемы $m, n \rightarrow \infty, a p_{k}, q_{k}, k=1,2, \ldots$, меняются так, чmo

$$
\begin{aligned}
& \mathbf{E} N(s, m, n)=m n D_{0} \rightarrow \lambda<\infty, \\
& m n D+s|H|(P+Q) \rightarrow 0 .
\end{aligned}
$$

Тогда

$$
\mathscr{L}(N(s, m, n)) \rightarrow \operatorname{Po}(\lambda)
$$

Если же

$$
m n D_{0} \rightarrow \infty, \quad D D_{0}^{-1}+s|H|(P+Q) \rightarrow 0,
$$

то функчия распределения случайной величинь

$$
\left(N(s, m, n)-m n D_{0}\right)\left(m n D_{0}\right)^{-1 / 2}
$$

сходится к стандартной нормальной функчии распределения.

Следующая теорема дает оценку близости распределения случайной величины $N(s, m, n)$ к сопровождающему пуассоновскому распределению. Обозначим через $\rho(F, G)$ расстояние по вариации между распределениями $F$ и $G$. Для распределений, сосредоточенных на $\{0,1, \ldots\}$, оно выражается формулой

$$
\rho(F, G)=\frac{1}{2} \sum_{k=0}^{\infty}|F\{k\}-G\{k\}| .
$$


Теорема 2. Пусть $m, n \geqslant 2 s-1$. Тогда

$$
\begin{aligned}
\left.\rho(\mathscr{L}(N(s, m, n))), \operatorname{Po}\left(m n D_{0}\right)\right) & \leqslant\left(1-e^{-m n D_{0}}\right)\left(2 m n D\left(m n D_{0}\right)^{-1}\right. \\
+ & \left.2(2 s-1)|H|(P+Q)+(2 s-1)^{2}\left(m n D_{0}\right)^{-1}|H|^{2} P Q\right) .
\end{aligned}
$$

Замечание 1. Можно рассматривать пары $H$-связанных цепочек в одной единственной последовательности $X$. Эти события будем называть $H$-повторениями. Число $M(s, m)$ $H$-повторений $s$-цепочек в последовательности $X$ выражается формулой

$$
M(s, m)=\sum_{1 \leqslant i<j \leqslant m} I\left(\exists h \in H:\left(h\left(X_{i}\right), \ldots, h\left(X_{i+s-1}\right)\right)=\left(X_{j}, \ldots, X_{j+s-1}\right)\right) .
$$

Можно показать, что при аналогичных предположениях предельные распределения случайных величин $N(s, m, n)$ и $M(s, m)$ совпадают. А именно, для числа $M(s, m)$ имеет место следующий аналог теоремы 1 (приводим его без доказательства).

Теорема 3. Пусть $m \rightarrow \infty, a p_{k}, k=1,2, \ldots$, меняются так, что

$$
\left(\begin{array}{c}
m \\
2
\end{array}\right) D_{0} \rightarrow \lambda<\infty, \quad m^{2} D+s|H| P \rightarrow 0
$$

с естественными изменениями в определениях $D_{0}$ и D. Тогда

$$
\mathscr{L}(M(s, m)) \rightarrow \operatorname{Po}(\lambda) .
$$

Если же

$$
\left(\begin{array}{c}
m \\
2
\end{array}\right) D_{0} \rightarrow \infty, \quad D D_{0}^{-1}+s|H| P \rightarrow 0
$$

то функция распределения случайной величины

$$
\left(M(s, m)-m^{2} D_{0} / 2\right)\left(m^{2} D_{0} / 2\right)^{-1 / 2}
$$

сходится к стандартной нормальной функции распределения.

Замечание 2. В работах С. М. Буравлева [2-4] и А. М. Шойтова [5, 6] рассматривалось отношение эквивалентности на $\mathbf{N}^{s}$ такое, что эквивалентными считаются цепочки, для которых найдется перестановка букв алфавита $\mathbf{N}$ из некоторого заданного подмножества $H=\left\{Z_{1}, \ldots, Z_{k}\right\}$ множества (группы) всех подстановок на $\mathbf{N}$, которая переводит одну цепочку в другую. В [5] такие цепочки были названы $H$-эквивалентными. При $H=\{e\}$ (напомним, что $e$ - тождественная подстановка), $H$-эквивалентными будут только совпадающие цепочки. Этот случай рассматривался в работах [8-10]. В работах [2-5] исследовалось число $\xi(H ; s, n)$ пар $H$-эквивалентных $s$-цепочек в последовательности $X=\left(X_{1}, \ldots, X_{m}\right)$ в случае, когда $H$ является набором из $k$ подстановок, нижние строки которых (будучи выписаны одна под другой) образуют латинский прямоугольник.

Замечание 3. Предположение о том, что $H$ является группой, необходимо для того, чтобы индуцируемое множеством подстановок $H$ отношение было отношением эквивалентности. 
Замечание 4. Из величин, участвующих в формулировках теорем 1 и 2 , наиболее сложно вычисляются $D_{0}$ и $D$. Чтобы обсудить способы их оценивания, рассмотрим некоторые частные случаи.

Пусть распределения $\left\{p_{k}\right\}$ и $\left\{q_{k}\right\}$ сосредоточены на множестве $\{1, \ldots, N\}$, причем распределение $\left\{q_{k}\right\}$ на нем равновероятно: $q_{1}=\ldots=q_{N}=1 / N$. Тогда

$$
D_{k} \leqslant \frac{|H|}{N^{s+k}}, \quad k=0,1, \ldots, s-1, \quad D<\frac{|H|}{N^{s}(N-1)} .
$$

Если при этом множество $H$ удовлетворяет условию

$$
|\{h \in H: h(x)=y\}| \leqslant 1, \quad x, y \in\{1, \ldots, N\},
$$

To

$$
D_{k}=|H| N^{-s-k}, \quad k=0,1, \ldots, s-1 .
$$

Используя эти свойства, из теоремы 1 нетрудно получить следующее утверждение.

Следствие 1. Пусть элементы последовательностей $X$ и Y распределены на множестве $\{1, \ldots, N\}$ равновероятно:

$$
p_{k}=q_{k}=1 / N, \quad k=1, \ldots, N,
$$

а множество Н удовлетворяет условию (5). Если

$$
\frac{m n|H|}{N^{s}} \rightarrow \lambda, \quad 0<\lambda<\infty, \quad \frac{s}{m}+\frac{s}{n} \rightarrow 0,
$$

mo

$$
\mathscr{L}(N(s, m, n)) \rightarrow \operatorname{Po}(\lambda) .
$$

Замечание 5. Последнее соотношение в (6) будет выполнено, если выполнено первое соотношение и

$$
\frac{\ln n}{m}+\frac{\ln m}{n}=o(\ln N) .
$$

Условие (7) является своего рода условием соразмерности длин последовательностей.

Вернемся к задаче об $H$-связанных цепочках. Каждому набору

$$
(i, j), \quad i=1,2, \ldots, m, \quad j=1,2, \ldots, n,
$$

сопоставим случайную величину

$$
W(i, j)=I(E(i, j)) .
$$

Тогда

$$
N(s, m, n)=\sum_{i=1}^{m} \sum_{j=1}^{n} W(i, j) .
$$

Обозначим через $W$ набор случайных величин $\{W(i, j)\}_{\Gamma}$ на множестве

$$
\Gamma=\{1, \ldots, m\} \times\{1, \ldots, n\} .
$$

Пусть $\Pi=\{\Pi(i, j)\}_{\Gamma}$ - набор независимых случайных величин, имеющих распределения Пуассона с параметрами $\mathrm{E}(i, j)=\mathrm{E} W(i, j)$. 
Теорема 4. Пусть параметры $m, n \geqslant 2 s-1$. Тогда

$$
\rho(\mathscr{L}(W), \mathscr{L}(\Pi)) \leqslant 2 m n D+2(2 s-1) \lambda|H|(P+Q)+(2 s-1)^{2}|H|^{2} P Q .
$$

Доказательство. Для элементов $(i, j) \in \Gamma$ будем использовать обозначение $a=(i, j)$. Рассмотрим множество

$$
\Gamma_{a}^{\text {ind }}=\left\{a^{\prime} \in \Gamma:\left|i^{\prime}-i\right| \geqslant s,\left|j^{\prime}-j\right| \geqslant s\right\} .
$$

Нетрудно проверить, что случайная величина $W(a)$ не зависит от совокупности случайных величин $\left\{W\left(a^{\prime}\right), a^{\prime} \in \Gamma_{a}^{\text {ind }}\right\}$. Положим

$$
\Gamma_{a}=(\Gamma \backslash\{a\}) \backslash \Gamma_{a}^{\text {ind }} .
$$

Воспользуемся теоремой 10.А из [11], из которой следует, что

$$
\rho(\mathscr{L}(W), \mathscr{L}(\Pi)) \leqslant \sum_{a \in \Gamma} \mathrm{E} W(a)(\mathrm{E} W(a)+\mathrm{E} U(a))+\sum_{a \in \Gamma} \mathrm{E} W(a) U(a),
$$

где

$$
U(a)=\sum_{a^{\prime} \in \Gamma_{a}} W\left(a^{\prime}\right) .
$$

Чтобы оценить первую сумму в правой части (12), заметим, что

$$
\left|\{a\} \cup \Gamma_{a}\right|=\left|\Gamma \backslash \Gamma_{a}^{\mathrm{ind}}\right|=m n-(m-2 s+1)(n-2 s+1) .
$$

Кроме того,

$$
\lambda(m, n)=m n \mathrm{E} W(a)=m n D_{0}, \quad m D_{0} \leqslant|H| P, \quad n R_{0} \leqslant|H| Q .
$$

Значит,

$$
\begin{aligned}
\sum_{a \in \Gamma} \operatorname{E} W(a)(\operatorname{E} W(a)+\operatorname{E} U(a)) & \leqslant(2 s-1) \lambda(m, n)^{2}\left(m^{-1}+n^{-1}\right) \\
& \leqslant(2 s-1) \lambda|H|(P+Q) .
\end{aligned}
$$

Оценим вторую сумму в правой части (12). Введем множества

$$
\begin{aligned}
\Gamma_{a}^{s} & =\left\{a^{\prime}=\left(i^{\prime}, j^{\prime}\right) \in \Gamma_{a}:-s<i^{\prime}-i=j^{\prime}-j<s\right\}, \\
\Gamma_{a}^{*} & =\Gamma_{a} \backslash \Gamma_{a}^{s}, \\
\Gamma_{a}^{* *} & =\left\{a^{\prime} \in \Gamma_{a}^{*}: 0<\left|i^{\prime}-i\right|<s, 0<\left|j^{\prime}-j\right|<s\right\} .
\end{aligned}
$$

Лемма 1. Пусть $a \in \Gamma$. При $a^{\prime} \in \Gamma_{a}^{*} \backslash \Gamma_{a}^{* *}$ выполнены неравенства

$$
\begin{array}{ll}
\mathbf{E} W(a) W\left(a^{\prime}\right) \leqslant|H| q^{s} \mathrm{E} W(a), & \text { если } 0<\left|i^{\prime}-i\right|<s, \\
\mathbf{E} W(a) W\left(a^{\prime}\right) \leqslant|H| p^{s} \mathrm{E} W(a), & \text { если } 0<\left|j^{\prime}-j\right|<s .
\end{array}
$$

При $a^{\prime} \in \Gamma_{a}^{* *}$ имеет место неравенство

$$
\mathrm{EW}(a) W\left(a^{\prime}\right) \leqslant|H|^{2} p^{s} q^{s} .
$$


Доказательство леммы 1 . Первое утверждение почти очевидно. Поэтому мы ограничимся доказательством второго утверждения.

Воспользуемся схемой доказательства леммы 2 из работы [8]. Выберем некоторые $a=(i, j) \in \Gamma$ и $a^{\prime}=\left(i^{\prime}, j^{\prime}\right) \in \Gamma_{a}^{* *}$. Рассмотрим двудольный граф $G=G\left(a, a^{\prime}\right)$ с множеством вершин

$$
\begin{aligned}
V & =V_{1} \cup V_{2}, \\
V_{1} & =\{i+1, \ldots, i+s-1\} \cup\left\{i^{\prime}+1, \ldots, i^{\prime}+s-1\right\}, \\
V_{2} & =\{j+1, \ldots, j+s-1\} \cup\left\{j^{\prime}+1, \ldots, j^{\prime}+s-1\right\},
\end{aligned}
$$

и множеством из $2 s$ ребер

$$
E=\left\{(i+k-1, j+k-1),\left(i^{\prime}+k-1, j^{\prime}+k-1\right): k=1, \ldots, s\right\} .
$$

Граф $G=G\left(a, a^{\prime}\right)$ не имеет циклов и состоит из связных компонент, представляющих собой простые цепи, в которых чередуются вершины из $V_{1}$ и $V_{2}$. Обозначим через $N_{s_{1}, s_{2}}$ число цепей с $s_{1}$ вершинами в $V_{1}$ и с $s_{2}$ вершинами в $V_{2},\left|s_{1}-s_{2}\right| \leqslant 1$. Цепь с $s_{1}$ вершинами в $V_{1}$ и с $s_{2}$ вершинами в $V_{2}$ имеет $s_{1}+s_{2}-1$ ребер, поэтому

$$
\sum_{s_{1}, s_{2}}\left(s_{1}+s_{2}-1\right) N_{s_{1}, s_{2}}=2 s .
$$

Докажем неравенство

$$
\mathbf{E} W(i, j ; h) W\left(i^{\prime}, j^{\prime} ; h^{\prime}\right)=\mathbf{P}\left\{E(i, j ; h) E\left(i^{\prime}, j^{\prime} ; h^{\prime}\right)\right\} \leqslant p^{s} q^{s} .
$$

Событие, указанное в (16), состоит в выполнении системы равенств

$$
Y_{j+k}=h\left(X_{i+k}\right), \quad Y_{j^{\prime}+k}=h^{\prime}\left(X_{i^{\prime}+k}\right), \quad k=1, \ldots, s .
$$

Равенства в этой системе можно разбить на группы равенств, каждая из которых соответствует своей цепи графа $G$. А именно, цепи

$$
\alpha_{1}, \beta_{1}, \ldots, \alpha_{r}, \beta_{r}, \alpha_{r+1}
$$

в графе $G$ (в этом случае $s_{1}=r+1, s_{2}=r$ ) отвечает набор равенств

$$
h\left(X_{\alpha_{k}}\right)=Y_{\beta_{k}}, \quad Y_{\beta_{k}}=h^{\prime}\left(X_{\alpha_{k+1}}\right), \quad k=1, \ldots, r .
$$

В этой системе значение $X_{\alpha_{1}}$ однозначно определяет остальные значения всех переменных системы. Поэтому вероятность выполнения этой системы равна

$$
\sum_{k=1}^{\infty} p_{k} q_{\phi_{1}(k)} \ldots q_{\phi_{2 r-1}} p_{\phi_{2 r}} \leqslant(p q)^{r} \sum_{k=1}^{\infty} p_{k}=(p q)^{\left(s_{1}+s_{2}-1\right) / 2} .
$$

Здесь использованы обозначения

$$
\phi_{1}=h, \quad \phi_{2}=h\left(h^{\prime}\right)^{-1}, \ldots, \phi_{2 r}=h\left(h^{\prime}\right)^{-1} \ldots h\left(h^{\prime}\right)^{-1} .
$$

Такая же оценка получается и для случая $s_{2}=s_{1}+1$. При $s_{1}=s_{2}=r$ для цепи вида

$$
\alpha_{1}, \beta_{1}, \ldots, \alpha_{r}, \beta_{r}
$$


получаем набор равенств, аналогичный набору (18), но без последнего равенства. В этом случае вероятность выполнения такого набора равенств равна

$$
\sum_{k=1}^{\infty} p_{k} q_{\phi_{1}(k)} \ldots q_{\phi_{2 r-1}} \leqslant(p q)^{r-1 / 2} \sum_{k=1}^{\infty} \sqrt{p_{k} q_{\phi_{1}(k)}} \leqslant(p q)^{\left(s_{1}+s_{2}-1\right) / 2}
$$

Аналогично рассматриваются цепочки, начинающиеся с $\beta_{1}$.

События, относящиеся к разным подсистемам (18), независимы. Поэтому вероятность выполнения системы (17) равна произведению вероятностей выполнения подсистем типа $(18)$, отвечающих цепям графа $G$. Поэтому в силу (15) получаем (16):

$$
\mathbf{P}\left\{E(i, j ; h) E\left(i^{\prime}, j^{\prime} ; h^{\prime}\right)\right\} \leqslant \prod_{s_{1}, s_{2}}(p q)^{\left(s_{1}+s_{2}-1\right) / 2}=p^{s} q^{s}
$$

Осталось воспользоваться очевидным неравенством

$$
\mathrm{E} W(i, j) W\left(i^{\prime}, j^{\prime}\right) \leqslant \sum_{h, h^{\prime} \in H} \mathrm{E} W(i, j ; h) W\left(i^{\prime}, j^{\prime} ; h^{\prime}\right)
$$

Лемма доказана.

Продолжим доказательство теоремы 4. Из определений следует неравенство

$$
\sum_{a \in \Gamma} \sum_{a^{\prime} \in \Gamma_{a}^{s}} \mathbf{E} W(a) W\left(a^{\prime}\right) \leqslant 2 m n\left(D_{1}+\ldots+D_{s-1}\right)=2 m n R .
$$

Используя лемму 1 , получаем, что

$$
\begin{gathered}
\sum_{a \in \Gamma} \sum_{a^{\prime} \in \Gamma_{a}^{*} \backslash \Gamma_{a}^{* *}} \operatorname{EW}(a) W\left(a^{\prime}\right) \leqslant(2 s-1)|H| \lambda\left(m p^{s}+n q^{s}\right), \\
\sum_{a \in \Gamma} \sum_{a^{\prime} \in \Gamma_{a}^{* *}} \operatorname{EW}(a) W\left(a^{\prime}\right) \leqslant(2 s-1)^{2}|H|^{2} m n p^{s} q^{s} .
\end{gathered}
$$

Подставив эти оценки вместе с (13) в (12), придем к неравенству (10). Теорема 4 доказана.

Теорема 2 доказывается аналогично, но ее доказательство опирается не на неравенство (12), а на неравенство

$$
\begin{aligned}
& \rho(\mathscr{L}(N(s, m, n))), \operatorname{Po}(\lambda(m, n))) \\
& \leqslant \frac{1-e^{-\lambda(m, n)}}{\lambda(m, n)}\left(\sum_{a \in \Gamma} \operatorname{E} W(a)(\operatorname{E} W(a)+\operatorname{E} U(a))+\sum_{a \in \Gamma} \operatorname{E} W(a) U(a)\right)
\end{aligned}
$$

(см. [11], теорема 1.A).

Теорема 1 является очевидным следствием теоремы 2. 


\section{Список литературы}

1. Буравлев С. М., Повторения с точностью до перестановок в последовательности независимых испытаний. Дискретная математика (1999) 11, №1, 53-75.

2. Буравлев С. М., Предельные теоремы для случайных величин от числа пар отрезков, связанных подстановками из латинского прямоугольника. Тезисы докл. Третьей Всероссийской школыколлоквиум по стохастическим методам. ТВП, Москва, 1996, 37-40.

3. Буравлев С. М., Локальная асимптотическая нормальность числа пар отрезков последовательности, связанных подстановками из латинского прямоугольника. Обозрение прикладной и промышленной математики (1998) 5, №2, 205-207.

4. Буравлев С. М., Уточнение одного предельного распределения. Обозрение прикладной и промышленной математики (1999) 6, №1, 127-128.

5. Шойтов А. М., Предельные распределения числа наборов $H$-эквивалентных отрезков в последовательности независимых испытаний. Обозрение прикладной и промышленной математики (1999) 6, №1, 218-220.

6. Шойтов А. М., Связь отрезков отношением $H$-эквивалентности в схеме серий. Обозрение прикладной и промышленной математики (1999) 6, №1, 220-221.

7. Михайлов В. Г., Предельная теорема Пуассона для числа $H$-совпадений цепочек. Обозрение прикладной и промышленной математики (2000) 7, №1, 123-124.

8. Зубков А. М., Михайлов В. Г., Предельные распределения случайных величин, связанных с длинными повторениями в последовательности независимых испытаний. Теория вероятностей и ее применения (1974) 19, №1, 173-181.

9. Зубков А. М., Михайлов В. Г., О повторениях $s$-цепочек в последовательности независимых величин. Теория вероятностей и ее применения (1979) 24, №2, 267-279.

10. Михайлов В. Г., Оценка точности сложной пуассоновской аппроксимации для распределения числа совпадающих цепочек. Теория вероятностей и ее применения (2001) 46, №4, 713-723.

11. Barbour A. D., Holst L., Janson S., Poisson approximation. Oxford University Press, Oxford, 1992.

Статья поступила 08.01.2002. 